\title{
Vivenciando a Seleção de Medicamentos em Hospital de Ensino
}

\author{
Experiencing Drug Selection in a Teaching \\ Hospital
}

\author{
Rachel Magarinos-Torres ${ }^{I}$ \\ Dante Pagnoncelli \\ Almiro Domiciano da Cruz Filho ${ }^{I I}$ \\ Claudia Garcia Serpa Osorio-de-Castro ${ }^{I I I}$
}

\section{PALAVRAS-CHAVE \\ - Medicamentos Essenciais. \\ - Uso de Medicamentos. \\ - Comitê de Farmácia e Terapêutica. \\ - Hospital.}

Recebido em: 11/06/2010

Reencaminhado em: 16/08/2010

Aprovado em: 26/08/2010

REVISTA BRASILEIRA DE EDUCAÇÃO MÉDICA
${ }^{I}$ Universidade Federal Fluminense, Niterói, RJ, Brasil; Fundação Oswaldo Cruz, Rio de Janeiro, RJ, Brasil.

II Fundação Oswaldo Cruz, Rio de Janeiro, RJ, Brasil.

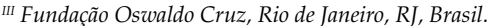

\section{RESUMO}

A seleção de medicamentos essenciais orienta a utilização eficaz, segura e racional de medicamentos. O texto descreve e discute o processo e os resultados alcançados pelo trabalho da Comissão de Farmácia e Terapêutica do Instituto Fernandes Figueira no momento de revisão de sua lista de medicamentos, ressaltando as implicações para a gestão, para a clínica e para a formação médica e da equipe multidisciplinar. Os dados trabalhados foram resgatados de documentos produzidos pelo grupo entre abril de 2005 e março de 2008. Foram trabalhados três grupos de medicamentos. Os achados apontam diminuição mínima de $44 \%$ no quantitativo de itens. O processo envolveu grande número de profissionais de diferentes categorias e setores do hospital. Embora demorada e complexa, estima-se que a revisão da lista de medicamentos tenha despertado todos para a necessidade de repensar a utilização do arsenal terapêutico, contribuindo para uma formação mais centrada no paradigma da evidência científica e favorecendo a adesão aos produtos finais.

Essential drug selection guides the efficacious, safe, and rational use of medicines. This article describes and discusses the process and results achieved in the work of the Pharmacy and Therapeutics Committee of the Fernandes Figueira Institute in Rio de Janeiro, Brazil, in the revision of its drug list, highlighting the implications for management, clinical practice, and medical and multidisciplinary team training. The data were retrieved from documents produced by the group from April 2005 to March 2008. Three drug groups were analyzed. The findings point to a decrease of at least $44 \%$ in the amount of items. The process involved a large number of staff members from different professions and departments in the hospital. Although long and complex, it is estimated that the revision of the drug list awakened the entire staff to the need for rethinking the use of the therapeutic armamentarium, contributing to training based on the scientific evidence paradigm and favoring adherence to the drugs on the final list. 


\section{INTRODUÇÃO}

A implantação de metodologias de avaliação de fármacos, aliada ao desenvolvimento da indústria farmacêutica a partir da década de 1950, trouxe a possibilidade de oferta de diferentes alternativas farmacoterapêuticas para enfrentamento das várias situações clínicas. Existem hoje, no mercado brasileiro, 2.034 medicamentos de referência sendo comercializados ${ }^{1}$. O número de fármacos para emprego no glaucoma, por exemplo, aumentou de 14 para 32 nos últimos 30 anos, ainda que substâncias utilizadas desde 1972 continuem mostrando resultados clínicos positivos².

Esta situação decorre mais da busca de aumento de competitividade por parte da indústria farmacêutica do que por demanda da clínica. Muitos dos novos produtos lançados são inovações incrementais, pequenas alterações na estrutura química de uma molécula já comercializada, que permitem ao fabricante, à custa de menor espaço de tempo e esforços em pesquisa e desenvolvimento, se manter ativo no mercado ${ }^{3}$. De 1998 a 2002, apenas 58 dos 415 medicamentos aprovados para comercialização nos Estados Unidos foram classificados como prováveis de representar aperfeiçoamento significativo em comparação com os produtos disponíveis ${ }^{4}$. Uma revisão de evidências conclui que, embora existam algumas diferenças farmacocinéticas, todos os inibidores da bomba de prótons possuem eficácia clínica similar ${ }^{5}$.

A propaganda direcionada a estudantes de Medicina, a prescritores e a consumidores finais fortalece a marca e garante o consumo mesmo quando da entrada de um concorrente ou de um genérico no mercado. Como consequência, existe uma inversão, e a oferta passa a induzir a demanda ${ }^{6}$. Por isto, o conceito de medicamentos essenciais, aqueles que satisfazem as necessidades prioritárias de saúde de determinada população, aparece nas estratégias traçadas tanto pela OMS como pelo Brasil ${ }^{7,8}$. A presença e a utilização de listas de medicamentos essenciais em diferentes níveis de atenção favorecem a promoção do uso racional de medicamentos ${ }^{9,10}$.

A formação médica não parece suficientemente voltada à apreciação do conceito de medicamentos essenciais, o que é paradoxal, tendo em vista a importância que os medicamentos assumem na terapêutica e na atenção ao paciente ${ }^{11,12}$. Em face deste problema, diversas ações educativas pautadas no conceito de medicamentos essenciais têm sido promovidas no Brasil. Em 2001, foi traduzido e adaptado para o português o Guia do Instrutor em Práticas da Boa Prescrição Médica, utilizado em cursos nacionais, regionais e estaduais sobre Ensino para o Uso Racional de Medicamentos, com vistas à capacitação de multiplicadores ${ }^{13}$.

A assistência farmacêutica é considerada parte integrante da política de saúde, visando garantir os princípios orientado- res do Sistema Único de Saúde (SUS). Um de seus eixos estratégicos é a adoção do conceito de medicamentos essenciais. A lista de medicamentos essenciais é composta por medicamentos escolhidos mediante aplicação de critérios definidos. A este processo denomina-se seleção de medicamentos. É atividade central da assistência farmacêutica e deve orientar todas as demais atividades relacionadas com medicamentos, tanto em espaços assistenciais como em sistemas de saúde ${ }^{3}$.

O processo de seleção envolve uma fase de preparação, com a constituição de um grupo multidisciplinar denominado genericamente Comissão de Farmácia e Terapêutica (CFT), uma etapa de escolha propriamente dita dos itens que irão compor a lista e um momento de divulgação e monitoramento dos resultados alcançados. A qualidade do processo está atrelada à composição da comissão. Dada a diversidade de competências exigidas, o grupo deve ser composto por diferentes categorias profissionais, como médicos, farmacêuticos e enfermeiros. Em hospitais de ensino, a CFT pode incluir residentes ${ }^{14}$.

A escolha dos itens que irão compor o subconjunto de medicamentos essenciais do hospital deve ser realizada com base em critérios de eficácia e segurança, seguidos de comodidade de utilização e custo. A qualidade da evidência clínica é refletida pela qualidade metodológica das pesquisas utilizadas na escolha comparativa dos medicamentos e, conjugada à vivência clínica, deve ser capaz de estimular a adesão dos médicos. A adoção deste paradigma desloca a prescrição da experiência individual, favorecendo decisões mais cientificas, eficazes e custo-efetivas ${ }^{14,15}$

O texto a seguir descreve e discute processo e resultados alcançados pelo trabalho da Comissão de Farmácia e Terapêutica do Instituto Fernandes Figueira (CFT/IFF). Traz também suas implicações para a gestão de medicamentos, para a clínica e para a formação médica e da equipe multidisciplinar. A experiência merece destaque por acontecer em um hospital polo de capacitação em Saúde da Criança, da Mulher e do Adolescente ${ }^{16}$. Além disto, embora o País conte com uma lista nacional de medicamentos essenciais, são poucos os hospitais que trabalham a seleção atendendo a critérios de qualidade, incluindo a existência de comissão multidisciplinar, capaz de contribuir na capacitação de médicos e residentes ${ }^{17,18}$.

\section{MÉTODO}

Desenvolveu-se um estudo de caso descritivo retrospectivo durante o primeiro semestre de 2008 com base na análise de documentos da Comissão de Farmácia e Terapêutica do Instituto Fernandes Figueira (CFT/IFF). O acesso a estes documentos foi formalmente autorizado por esta Comissão e pela Direção Ge- 
ral da unidade, mediante compromisso de sigilo e confidencialidade quanto à identificação e dados pessoais dos envolvidos.

O Instituto Fernandes Figueira (IFF) é a Unidade da Fundação Oswaldo Cruz (Fiocruz) direcionada à atenção materno-infantil de alta complexidade. É referência nacional em genética médica, perinatologia, cirurgia infantil, doenças pediátricas, endometriose e gestação de alto risco. Em 2007, esta unidade, situada no Rio de Janeiro, atendeu cerca de 90 mil pacientes em consultas ambulatoriais e hospitalizações. Além da assistência direta, o IFF assume a missão de contribuir com pesquisa, ensino, desenvolvimento tecnológico e extensão no âmbito da saúde da mulher, da criança e do adolescente ${ }^{16}$.

Este estudo considerou documentos relacionados à CFT/ IFF produzidos entre abril de 2005 e março de 2008, a saber: as atas de reuniões, as mensagens eletrônicas, as listas e suplementos do formulário terapêutico construídos ao longo desse período $^{19-21}$

O conteúdo resgatado foi alocado em três categorias de análise, enunciadas a priori, com base no referencial teórico da seleção de medicamentos: (a) organização, composição e fluxo de trabalho da comissão responsável pelo processo; (b) seleção e revisão per se; (c) produtos da seleção. Os resultados foram confrontados com a literatura.

\section{RESULTADOS E DISCUSSÃO}

Em 1996, foi implantada a primeira Comissão de Farmácia e Terapêutica do Instituto Fernandes Figueira (CFT/IFF). O processo deu origem a uma lista de medicamentos que foi publicada em 1997. De 1997 a 2001, a CFT/IFF manteve atividade, mas a revisão completa da lista não ocorreu. Os registros pertinentes a esse período foram perdidos em sinistro ocorrido no dia 8 de abril de 2008, inviabilizando a consulta.

Entre 2001 e 2005, a CFT/IFF esteve desativada, permanecendo a lista de 1997 teoricamente vigente, ainda que com inúmeras adições e alterações incorporadas sem processo técnico específico. Em abril de 2005, a CFT/IFF foi reativada e teve início o processo de revisão da seleção de medicamentos no hospital.

\section{Questões organizacionais, composição e fluxo de trabalho}

A revitalização da CFT/IFF, em junho de 2005, aconteceu em decorrência da articulação de dois médicos e um farmacêutico da instituição. Este grupo, em contato direto com representantes dos principais setores do hospital, formou a CFT/ IFF. O nome dos interessados foi encaminhado à Direção, que nomeou os membros por portaria interna.

Embora não tenham sido identificados os motivos exatos que levaram à mobilização dos profissionais, os documentos consultados e a literatura apontam algumas possibilidades: ampliar o número de medicamentos disponíveis, racionalizar a oferta dos medicamentos ou garantir, estrategicamente, insumos para seu trabalho.

A clínica pode, efetivamente, demandar mais ou melhores opções terapêuticas, tendo em vista a complexidade do cuidado oferecido e o perfil da população atendida. Este é um axioma dentro do conceito de medicamentos essenciais, uma vez que a essencialidade é relativa ${ }^{3}$. Ampliar o número de medicamentos oferecidos pode, portanto, contar com justificativa não apenas quanto à adequação, mas quanto à necessidade.

Por outro lado, os prescritores são suscetíveis a estratégias da indústria para forçar uma ampliação da demanda. A relação destes com a indústria farmacêutica tem início ainda na graduação. $\mathrm{O}$ assédio da indústria se dá no sentido de oferecer, por meio de representantes, informação supostamente acurada sobre os medicamentos e as alternativas existentes. Este vínculo é fortalecido durante a residência e a vida profissional com as demais estratégias promocionais, como a distribuição de material publicitário e prêmios ${ }^{22}$. O residente tende a assumir postura semelhante à do preceptor médico, em espaço de treinamento e formação, com aprendizado centrado no modo vigente de fazer, na rotina do serviço. Neste sentido, chamam atenção os achados de Fagundes et al. ${ }^{23}$, citando que $98 \%$ dos médicos são visitados com frequência por propagandistas, $22 \%$ confiam plenamente nas peças publicitárias distribuídas pelos laboratórios e $14 \%$ afirmam prescrever em função do recebimento de prêmios. Esta situação é negativa para os pacientes; a maioria das peças publicitárias omite dados importantes, como contraindicações, efeitos adversos e interações ${ }^{24}$.

Maior conhecimento sobre riscos relacionados à terapêutica poderia ajudar a reforçar a preocupação dos prescritores com as consequências da presença de itens desnecessários à segurança dos pacientes e dos processos de trabalho. $\mathrm{O}$ fato de não haver divulgação da incidência e gravidade de eventos adversos relacionados com medicamentos na unidade fragiliza o processo de assistência. Embora a divulgação seja um indicativo da qualidade hospitalar ${ }^{17}$ e o IFF seja classificado pelo Ministério da Saúde como hospital sentinela ${ }^{25}$, o acompanhamento da segurança dos medicamentos mediante atividades rotineiras de farmacovigilância parece não ocorrer. O IFF não difere em nada do padrão nacional, já que ocorre farmacovigilância em apenas $0,4 \%$ dos hospitais brasileiros ${ }^{17}$.

A favor da segunda possibilidade, pesa a presença do farmacêutico no grupo inicial. Este profissional recebeu conteúdo teórico relacionado à seleção de medicamentos durante a graduação ${ }^{26}$, possui posição privilegiada para dimensionar o quantitativo de itens disponíveis no hospital e exerce ativi- 
dades gerenciais e clínicas que são favorecidas pela racionalização da oferta ${ }^{3}$. O trabalho em equipe favorece a troca de conhecimentos e, neste caso, assume importância na disseminação do paradigma das condutas baseadas em evidências ${ }^{14}$.

O desabastecimento é uma situação corriqueiramente vivenciada por gestores e profissionais de saúde em instituições públicas. O receio quanto à falta medicamentos pode também ter impulsionado o movimento de seleção pelos profissionais. A diminuição do número de itens é percebida como uma estratégia para garantir que os prioritários não faltem. Reis e Perini ${ }^{27}$ ressaltam o enorme potencial de contribuição de uma comissão de farmácia e terapêutica e de um processo de seleção de qualidade no gerenciamento do desabastecimento de medicamentos em serviços de saúde.

A participação na CFT/IFF pode ter sido também uma estratégia para garantir na lista a prioridade de inclusão dos medicamentos demandados pelo próprio profissional ou departamento. Considerando a estrutura organizacional segmentada, característica dos hospitais ${ }^{28}$, esta é uma postura individualista que pouco contribui para o coletivo da instituição.

O processo de trabalho de revisão da seleção de medicamentos no IFF foi mediado por reuniões presenciais, seguido de discussões virtuais. Além disto, parte da comissão atuou com dedicação exclusiva a esta finalidade por 20 horas semanais. Os relatos apontam a dificuldade de estabelecer um espaço físico fixo para as reuniões e para o grupo.

Durante o período observado, houve 73 reuniões, distribuídas em: 20 a partir de abril de 2005, 22 em 2006, 26 em 2007 e 5 até março de 2008. Foram recuperadas 69 atas. Os registros permitiram obter informação do tempo de duração de 58 encontros, totalizando 84,78 horas de trabalho. Em média, as reuniões duraram cerca de 1 hora e 45 minutos cada.

Participaram destes encontros 29 pessoas, 13 como membros efetivos da CFT/IFF, 13 na posição de consultores e quatro estudantes de graduação em Farmácia. O número médio de participantes por reunião foi 5,9. Foi possível observar a existência de um núcleo regular de cinco membros efetivos com presença em mais de $60 \%$ das reuniões e cujas faltas aparecem dispersas ao longo do período observado. Integraram este grupo três médicos e dois farmacêuticos. Os demais tiveram participação pontual.

Os consultores foram médicos e enfermeiros do hospital. Como "especialistas colaboradores", aportaram conhecimentos teóricos pertinentes a sua área de atuação e condicionantes do trabalho na instituição. A cada um foi fornecida uma declaração de participação e a inclusão de seu nome no formulário terapêutico. Não houve nenhum caso de desistência no decorrer dos trabalhos.
De todos os participantes, independentemente da posição na CFT/IFF ou da categoria profissional, foi exigida declaração de ausência de conflito de interesses. O modelo de termo foi adaptado daquele utilizado pela Comissão Técnica e Multidisciplinar de Atualização da Relação Nacional de Medicamentos Essenciais ${ }^{29}$. Esta atitude encontra respaldo nos princípios fundamentais do Código de Ética Médica. Segundo este documento, "Quando envolvido na produção de conhecimento científico, o médico agirá com isenção e independência, visando ao maior benefício para os pacientes e a sociedade." ${ }^{\prime 30}$ (capítulo I, inciso XXIII). "É vedado ao médico [...] exercer a profissão com interação ou dependência de farmácia, indústria farmacêutica, óptica ou qualquer organização destinada à fabricação, manipulação, promoção ou comercialização de produtos de prescrição médica, qualquer que seja sua natureza." ${ }^{30}$ (capítulo VIII, artigo 68). A declaração de ausência de conflito diminui o viés mercadológico no processo de seleção, estimula a reflexão acerca do comportamento médico e, consequentemente, pode atuar na utilização de evidência enviesada por interesses comerciais.

Em 2006, teve início o trabalho a distância com auxílio de um ambiente virtual colaborativo gratuito, externo à instituição, composto de um grupo de correio eletrônico e um repositório de dados. Somente os membros efetivos tinham acesso a este instrumento. Foram localizadas 856 mensagens, em sua maioria apresentando as atas de reunião e troca de informações para resolução de pendências destacadas nas reuniões. A dinâmica focada parece ter sido responsável pela boa avaliação apontada pelos profissionais, levando à continuidade do uso desta forma de trabalho. Bejarano et al. ${ }^{31}$, concluíram que os principais problemas de comunicação com a utilização desta tecnologia são decorrentes do não estabelecimento de regras quanto ao uso e envio de material e da falta de um calendário ou agenda de trabalho comum. O número de reuniões presenciais é apontado como um fator decisivo para o bom andamento do trabalho virtual.

Comparando a CFT/IFF com o padrão recomendado pela $\mathrm{OMS}^{14}$, houve atendimento parcial aos indicadores propostos. Os resultados positivos da CFT/IFF incluem: a representatividade dos profissionais e setores da instituição, a composição multidisciplinar, a ausência de conflito de interesses dos membros, a periodicidade das reuniões e a documentação dos processos e das decisões. Em contrapartida, mesmo tendo sido constituída pela Direção, a CFT/IFF não aparece no organograma do hospital, conforme indicado no guia publicado pela OMS.

Quando observada frente a outros hospitais, a CFT/IFF trabalhou com um número pequeno de membros efetivos e 
teve quase o dobro de reuniões presenciais por ano. Os outros parâmetros não diferiram. Mannebach et al. ${ }^{32}$, a partir de questionário enviado para 267 hospitais universitários americanos, encontraram, com taxa de resposta de 70\%, que, em média, as CFT possuem 19,3 membros. A maior parte dos integrantes são médicos e farmacêuticos. O número médio de encontros anuais ficou em torno de 9,7, e o tempo médio de duração da reunião foi de cerca de 1,3 horas. Apenas 5\% dos grupos utilizavam correio eletrônico.

A carência de relatos de experiências brasileiras no tema pode estar associada ao maior número de reuniões, pois o grupo teve de "aprender a fazer" 33 . Soma-se a isto o pouco apoio legal para o estabelecimento de CFT no Brasil. Os hospitais brasileiros não são obrigados legalmente a ter uma CFT, como acontece com outras instâncias de trabalho interdisciplinar, a exemplo da comissão de controle de infecção hospitalar. Países como Alemanha e a Suécia possuem normativas com este teor ${ }^{18}$. O apoio legal é capaz de conferir a necessária autoridade executiva, facilitar a obtenção de colaboração dos demais profissionais da organização e viabilizar os recursos físicos necessários, incluindo o financiamento ${ }^{28}$.

Oito membros efetivos se integraram ou se desligaram da CFT/IFF em algum momento do período observado. A variabilidade de componentes pode ter relação com a dificuldade de realização de trabalho interdisciplinar em organizações de saúde. A autonomia, fruto do alto grau de especialização dos profissionais envolvidos, segmenta o espaço em distintos grupos de interesses e dificulta o trabalho conjunto ${ }^{28,33}$. A especialização precoce, o movimento de escolha da especialidade médica ainda durante a graduação, tem resultado em lacunas na formação geral do médico e pouco contribuído para o reconhecimento do trabalho multidisciplinar e a valorização de processos que perpassam diversos setores e espaços do hospital ${ }^{34}$.

\section{O Processo de Seleção de Medicamentos no IFF}

O processo de revisão da seleção de medicamentos teve início com consulta aberta aos profissionais do hospital. As atividades subsequentes foram cíclicas e envolveram organização dos dados, busca de evidências, discussão entre os membros efetivos da CFT/IFF, participação de consultores, redação final para determinado grupo terapêutico, da lista e do formulário terapêutico.

Com base em modelos utilizados por outros hospitais brasileiros ${ }^{35,36}$, foram desenvolvidos instrumentos para participação e sensibilização dos profissionais que atuam no hospital. O Formulário de Sugestões de Atualização da Lista de Medicamentos Padronizados do IFF foi encaminhado a todos os departamentos, setores e serviços, junto com a lista de 1997.
Além disto, foram afixados cartazes e distribuídos folhetos sobre a importância da participação para a qualidade do produto final e da relevância deste para a instituição. A preocupação em envolver o maior número de profissionais, direta ou indiretamente, relaciona-se à disseminação da importância e dos conceitos que embasam o processo, à legitimação da lista e à garantia de adesão aos produtos. Estudos internacionais destacam esta baixa adesão como um problema do processo de seleção em hospitais. O mesmo parece acontecer em outros níveis organizacionais, como sistemas de saúde ou níveis de gestão ${ }^{37,38}$.

Findo o prazo, os membros avaliaram que o retorno não ocorreu de acordo com as expectativas. Estimou-se que um maior número de pessoas, muitas das quais importantes membros do corpo clínico, poderia contribuir. Para contornar este problema, a CFT/IFF dividiu Departamentos, Setores e Serviços do IFF entre seus membros, chamados nesse momento de "supervisores", e lhes atribuiu a tarefa de mobilizar seus pares via contato direto. O contato direto e a valorização dos atores internos têm papel fundamental na liderança em organizações reguladas pela qualificação dos profissionais, como são os hospitais ${ }^{28}$.

Em seguida, foi construída uma planilha (aplicativo Excel, Microsoft Corp.) com as variáveis: nome do medicamento segundo Denominação Comum Brasileira (DCB), concentração, forma farmacêutica, presença na lista de 1997, uso no IFF, origem e justificativa da solicitação. Compuseram a planilha 610 itens. Medicamentos com a mesma DCB, mas diferindo em concentração, forma farmacêutica e/ou apresentação, foram considerados separadamente. No sentido de favorecer uma análise comparativa, a CFT/IFF subdividiu a relação utilizando a classificação Anatomical Therapeutic Chemical (ATC) da $\mathrm{OMS}^{39}$. Como resultado, 12 grupos com variação de 10 a 147 itens foram formados. A ordem de análise dos grupos foi definida pelo maior número de itens, menor dispersão de utilização no hospital e maior facilidade de comunicação entre os membros da CFT/IFF e os setores que utilizavam os medicamentos. Esta diferiu de outras listas de medicamentos essenciais, em que, geralmente, se obedece a prioridades, com base na obsolescência das evidências que substanciam as escolhas ${ }^{26,35}$. Como a lista do IFF estava desatualizada havia muito tempo, possivelmente partiu-se da premissa de que todos os itens eram merecedores de análise.

Grupo a grupo e para cada medicamento, foram pesquisadas as indicações clínicas, evidências de eficácia e segurança, disponibilidade no mercado nacional, uso no IFF e preço unitário, considerando a apresentação. A busca foi realizada por estudantes do último período de Farmácia sob supervisão 
de um dos membros e envolveu consulta à Relação Nacional de Medicamentos Essenciais (Rename) ${ }^{40}$, ao Formulário Terapêutico da $\mathrm{OMS}^{41}$, ao banco de dados Micromedex Healthcare Series $^{42}$, ao Bulário Eletrônico da Agência Nacional de Vigilância Sanitária ${ }^{43}$, ao Banco de Preços do Ministério da Saúde $(\mathrm{BPS})^{44}$, ao Dicionário de Especialidades Farmacêuticas (DEF) ${ }^{45}$ e à Revista ABCFARMA ${ }^{46}$. As duas últimas fontes foram utilizadas para verificar a disponibilidade no mercado nacional e servir como referencial de preço quando este inexistia no BPS.

A utilização do Bulário ${ }^{43}$ requer considerações. As informações contidas nesta fonte são provenientes da indústria farmacêutica e, portanto, devem ser utilizadas com cautela. Todavia, é possível encontrar ali a indicação terapêutica para a qual o medicamento possui registro, o que pode ser interessante do ponto de vista de inibir o uso off label, principalmente em hospital materno-infantil. Carvalho et al. ${ }^{47}$ identificaram alta prevalência de medicamentos off label na UTI pediátrica de um hospital público brasileiro com atividades voltadas para ensino e pesquisa.

Houve dificuldade de obtenção do custo considerando o tratamento-dia. Vários medicamentos são utilizados em mais de um protocolo. Ainda que possivelmente conhecidos pelas equipes de cuidado, os protocolos não eram divulgados e não estavam disponíveis para a CFT/IFF. A aplicação não transparente de protocolos clínicos contraria a boa prática, dificulta a construção de demanda com impacto na continuidade de abastecimento e aumenta os riscos para quem manipula ${ }^{47}$.

A etapa de análise comparativa priorizou a identificação de pontos críticos por grupo, como efetividade no hospital, existência de duplicidade de recurso farmacoterapêutico para uma mesma situação clínica, exigências específicas da legislação brasileira e problemas relativos às práticas de utilização. De acordo com a necessidade, foram envolvidos os especialistas colaboradores. A CFT/IFF optou por debater as questões enunciadas com os consultores durante reunião presencial, e o conteúdo foi registrado.

Os mesmos dados que embasaram a construção da lista foram utilizados na confecção do formulário terapêutico. Não houve coleta de evidências específica para compor o formulário. A versão inicial do texto foi encaminhada aos consultores para revisão final. Feitas as devidas alterações, o documento foi impresso e distribuído gratuitamente aos setores do hospital. A tiragem, diferentemente do recomendado, foi definida pela disponibilidade financeira. $\mathrm{O}$ indicado é que cada profissional de saúde que atue no hospital receba um exemplar do formulário ${ }^{48}$.

O formulário terapêutico é um importante instrumento na promoção do uso racional. Quando a lista é divulgada junto com informações farmacoterapêuticas e com orientações administrativas, diminuem-se as chances de eventos adversos. A opção por analisar um subgrupo de medicamentos, elaborar a lista e logo em seguida construir o respectivo formulário parece favorecer a participação dos consultores. A proximidade entre o momento do encaminhamento das sugestões de inclusão ou exclusão e a resposta, ainda que seja uma lista parcial, gera credibilidade ao processo. Ademais, quando publicado em suplemento, o formulário tem seu tamanho reduzido, o que facilita o manejo e, possivelmente, a adesão às recomendações. Em contrapartida, a escolha desta forma de trabalho pode levar à perda de uniformidade e a atrasos na definição da lista completa de medicamentos essenciais ${ }^{48}$.

\section{Os Produtos da Seleção}

Em 2005, 539 itens tinham registro de movimentação no Serviço de Farmácia. A seleção de 1997 relacionou 252 itens. Isto significa que, durante os oito anos sem revisão, foram incorporados 287 itens à lista, o que, percentualmente, significa incremento superior a $100 \%$. Esse fato questiona a racionalidade da incorporação de medicamentos sem análise técnica detalhada e confirma a importância da existência de uma comissão de farmácia de caráter permanente ${ }^{3}$.

Observando os grupos revisados até março de 2008, a CFT/IFF analisou 16 itens do Grupo L - agentes antineoplásicos e imunomoduladores, 36 do N01 - anestésicos, 9 do N02 - analgésicos e 37 do R - medicamentos com atuação no sistema respiratório. $\mathrm{O}$ grupo $\mathrm{N}$ foi subdividido em três subgrupos e dois deles revisados. Do conjunto final de 98 itens analisados no período, 33\% estavam presentes na lista de 1997, 48\% foram incorporados acriticamente entre 1997 e 2005, e 19\% foram solicitações de inclusão. Dos 98, 45 itens permaneceram na lista. Foram acatadas 9 das 19 solicitações de inclusão recebidas. Houve diminuição mínima de $44 \%$ dos itens, tanto no somatório total como em cada um dos quatro grupos trabalhados.

A diminuição no número de itens, por si só, é um ganho para a assistência farmacêutica hospitalar. A logística, o fornecimento de informação e o acompanhamento do uso pela farmácia hospitalar são favorecidos quando há um número menor de medicamentos disponíveis. Além disso, os gastos totais tendem a diminuir com o aumento do volume de compra de um mesmo item, por economia de escala. O montante financeiro requerido pode comprometer o tratamento do conjunto de pacientes ${ }^{3,27}$.

Os pacientes, por sua vez, ficam mais expostos a eventos adversos, tanto do tipo erro de medicação, em decorrência de erros de processo pela variedade de itens circulantes, como 
do tipo efeito adverso, em virtude da exposição a mais medicamentos com limitadas informações sobre sua segurança. $\mathrm{O}$ tempo de uso de um medicamento é diretamente proporcional ao conhecimento sobre ele. Os ensaios clínicos e estudos epidemiológicos apresentados no momento de solicitação de registro do produto não esgotam as possibilidades de detecção desses efeitos ${ }^{49}$.

Em alguns momentos, o critério decisivo na escolha dos medicamentos foi o processo de trabalho existente no IFF. A lista de medicamentos, como produto final da seleção, deve ter relação direta com o perfil de atendimento e estar de acordo com as possibilidades estruturais da organização ${ }^{14}$. A não inclusão de antineoplásicos no grupo L01 ocorreu, por exemplo, em decorrência de ausência de condições físicas no hospital para manipulação segundo as recomendações da Agência Nacional de Vigilância Sanitária ${ }^{50}$. A CFT/IFF apontou que não teria sentido estruturar o Serviço de Farmácia para manipulá-los, frente à pequena demanda.

Outro aspecto que cabe exemplificar é a influência da fonte financiadora no processo de seleção. Tanto a goserelina como a leuprolida possuem a mesma indicação clínica e ambas integram o Componente de Medicamentos de Dispensação Excepcional, sendo fornecidas pela instância estadual sem custos diretos para o hospital. Isto poderia desestimular a escolha baseada em evidências, fato que não ocorreu. A decisão pela leuprolida se apoiou na necessidade de atenção especial na administração da goserelina, no melhor perfil de segurança da leuprolida em crianças e no seu custo/dose ${ }^{51}$.

Foram produzidos, no período observado, três suplementos do formulário terapêutico. O formulário de um dos grupos analisado estava na etapa de revisão final em março de 2008. A proximidade com os consultores possibilitou definir indicações de uso no IFF e pactuar normas de procedimento. Em todos os três, os medicamentos estão vinculados à indicação. Um dos suplementos traz protocolo simplificado de todos os medicamentos, relacionando diagnóstico, medicamentos, posologia, tempo de uso e manejos específicos.

\section{CONSIDERAÇÕES FINAIS}

A seleção é um processo complexo. A simples exclusão ou inclusão de medicamentos sem análise crítica não possibilita construir uma lista de medicamentos essenciais. A avaliação comparativa com base em evidências científicas de qualidade, acrescida do perfil de utilização e de ponderação quanto aos gastos envolvidos, é inerente ao conceito de seleção de medicamentos.

Além de complexa, a seleção deve ser legitimada em um processo participativo. Em 36 meses, a CFT/IFF concluiu a seleção de 98 (16\%) medicamentos dos 610 a analisar. O tempo gasto pela atividade pode ter refletido o cuidado com o processo, mas, por outro lado, trouxe desgaste. A falta de experiência, a carência de relatos de experiência na literatura e a opção pelo formato participativo do processo podem ser imputadas como grandes responsáveis pela duração da revisão. Caso o processo tivesse caminhado mais rapidamente, poderiam ter sido examinados resultados adicionais, como o nível de adesão aos produtos da lista.

Este trabalho expôs a revisão da lista de medicamentos essenciais em um hospital brasileiro, no caso, o IFF. O recorte metodológico permitiu investigar o objetivo desenhado, uma vez que, teoricamente, o processo de revisão da lista inclui todas as etapas da seleção de medicamentos, ponderando aspectos gerenciais e clínicos. No entanto, entrevistas individuais e confidenciais com os participantes e com os residentes poderiam ter permitido um aprofundamento maior quanto às questões relativas ao processo e aos ganhos para a formação médica. Em contrapartida, a inclusão de um grande número de profissionais do hospital integrou setores e estima-se que o processo os tenha despertado para a necessidade de repensar a utilização do arsenal terapêutico, favorecendo a adesão aos produtos finais por prescritores e residentes.

\section{REFERÊNCIAS}

1. Agencia Nacional de Vigilância Sanitária. Lista de medicamentos de referência [online]. [acesso em 23 jan. 2009]. Disponível em: http://www.anvisa.gov.br/medicamentos/referencia/index.htm.

2. Souza Filho JP, Dias ABT, Lima FAAS, Sartori MF, Martins MC. A evolução do mercado farmacêutico brasileiro no tratamento do glaucoma nos últimos 30 anos. Arq Bras Oftalmol. 2003;66(6):811-7.

3. Oliveira MA, Bermudez JAZ, Osorio-De-Castro, CGS. Assistência Farmacêutica e Acesso a Medicamentos. Coleção Temas em Saúde. Rio de Janeiro: Ed. Fiocruz; 2007.

4. Angel M. A verdade sobre os laboratórios farmacêuticos. Rio de Janeiro: Record; 2007.

5. Wannmacher L. Inibidores da bomba de prótons: indicações racionais. Uso Racional de Medicamentos: Temas selecionados OPAS. 2004;2(1):1-5.

6. Gadelha CAG, Quental C, Fialho BC. Saúde e inovação: uma abordagem sistêmica das indústrias da saúde. Cad Saúde Pública. 2003;19(1):47-59.

7. World Health Organization. WHO Medicines Strategy 2008-2013. Draft, version 6, 2008. [acesso em 26 fev. 2009]. Disponível em http://www.who.int/medicines/areas/ policy/medstrategy_consultation/en/index.html. 
8. Ministério da Saúde. Secretaria de Ciência, Tecnologia e Insumos Estratégicos. Departamento de Ciência e Tecnologia. Agenda nacional de prioridades de pesquisa em saúde. Brasília: MS; 2008. (Série B. Textos Básicos em Saúde)

9. World Health Organization. Policy Perspectives on Medicines: the Selection of Essential Medicines. Geneva; 2002.

10. Brasil. Ministério da Saúde. Portaria GM nº 3916 de 30 de outubro de 1998. Aprova a Política Nacional de Medicamentos. Diário Oficial da União. Brasília, 10 nov 1998, seção 1 p18.

11. Santos V, Nitrini SMOO. Indicadores do uso de medicamentos prescritos e de assistência ao paciente de serviços de saúde. Rev Saúde Pública. 2004;38(6):819-34.

12. Dal Pizzol TS, Trevisol DJ, Heineck I, Flores LM, Camargo AL, Köenig A et al. Adesão a listas de medicamentos essenciais em municípios de três estados brasileiros. Cad Saúde Pública. 2010;26(4):827-36.

13. Organização Mundial da Saúde. Departamento de Medicamentos Essenciais e Política de Medicamentos. Guia do Instrutor em Práticas da Boa Prescrição Médica. Tradução e adaptação para o português: Luiza VL e Osorio de Castro CGS. [S.1.]:OMS; 2001.

14. World Health Organization. Drug and therapeutics committees: a practical guide Department of Essential Drugs and Medicines Policy. Geneva: WHO; 2003.

15. Sacket DL, Rosenberg WC, Gray JAM, Haynes RB, Richardson WS. Evidence based medicine: what it is and what it isn't. BMJ. 1996;312:71-2.

16. Fundação Oswaldo Cruz. Relatório de Atividades de 2007. Rio de Janeiro: Ed. Fiocruz; 2008.

17. Osorio-de-Castro CGS, Castilho SR, org. Diagnóstico da Farmácia Hospitalar no Brasil. Rio de Janeiro: Ed. Fiocruz; 2004.

18. Marques DC, Zucchi P. Comissões farmacoterapêuticas no Brasil: aquém das diretrizes internacionais. Rev Panam Salud Publica. 2006;19(1):58-63.

19. Comissão de Farmácia e Terapêutica do Instituto Fernandes Figueira. Formulário Farmacoterapêutico 2008: suplemento antineoplásicos Grupo L. Rio de Janeiro: Ed. Fiocruz; 2008.

20. Comissão de Farmácia e Terapêutica do Instituto Fernandes Figueira. Formulário Farmacoterapêutico 2008: suplemento analgésicos Grupo N01. Rio de Janeiro: Ed. Fiocruz; 2008.

21. Comissão de Farmácia e Terapêutica do Instituto Fernandes Figueira. Formulário Farmacoterapêutico 2008: suplemento anestésicos Grupo N02. Rio de Janeiro: Ed. Fiocruz; 2008.
22. Palácios M, Rego S, Lino MH. Promoção e propaganda de medicamentos em ambientes de ensino: elementos para o debate. Interface Comum Saúde Educ. 2008;12(27):893-905.

23. Fagundes MJD, Soares MGA, Diniz NM, Pires JR, Garrafa V. Análise bioética da propaganda e publicidade de medicamentos. Ciênc Saúde Colet. 2007;12(1):221-9.

24. Barros JAC, Joany S. Anúncios de medicamentos em revistas médicas: ajudando a promover a boa prescrição? Ciênc Saúde Colet. 2002;7(4):891-8.

25. Agência Nacional de Vigilância Sanitária. Mapa de Localização dos Hospitais Sentinelas. Região Sudeste - Rio de Janeiro [online]. [acesso em 10 fev. 2009]. Disponível em http:/ / www.anvisa.gov.br/farmacovigilancia/oficinas/ mapa/rio.htm.

26. Brasil. Ministério da Educação. Conselho Nacional de Educação. Câmara de Educação Superior. Resolução CNE/CES de 19 de fevereiro de 2002. Institui diretrizes curriculares nacionais do curso de graduação em farmácia. Diário Oficial da União. Brasília, 4 mar. 2002; Seção 1, p. 9.

27. Reis AMM, Perini E. Desabastecimento de medicamentos: determinantes, conseqüências e gerenciamento. Ciênc Saúde Colet. 2008;13(supl):603-10.

28. Mendes EV. Os sistemas de serviços de saúde; o que os gestores deveriam saber sobre essas organizações complexas. Fortaleza: Escola de Saúde Pública do Ceará; 2002.

29. Ministério da Saúde. Secretaria de Ciência, Tecnologia e Insumos Estratégicos. Departamento de Assistência Farmacêutica e Insumos Estratégicos. Relação Nacional de Medicamentos Essenciais. Brasília: Ed MS; 2008.

30. Conselho Federal de Medicina. Código de Ética Médica. Resolução CFM 1931 de 17 de setembro de 2009. Diário Oficial da União. Brasília 13 abr 2010, Seção 1, p. 173.

31. Bejarano VC, Pilatti LA, Scandelari L, Oliveira AC . Equipes virtuais: um estudo de caso na indústria têxtil norte-americana. Prod. 2006;16(1):161-70.

32. Mannebach MA, Ascione FJ, Gaither CA, Bagozzi RP, Cohen IA, Ryan ML. Activities, functions, and structure of pharmacy and therapeutics committees in large teaching hospitals. Am J Health Syst Pharm. 1999;56(7):622-8.

33. Silva NEK, Oliveira LA, Figueiredo WS, Landroni MAS, Waldeman CCS, Ayres JR. Limites do trabalho multiprofissional: estudo de caso dos centros de referência para DST/ Aids. Rev Saúde Pública. 2002;36(4):108-16.

34. Ribeiro RC, Fonseca-Guedes CHF, Nunes, MPT. Médicos recém-formados: sólida formação geral ou sólida formação especializada?. Rev Bras Educ Med. 2009;33(4):571-85.

35. Hospital das Clínicas da Faculdade de Medicina da Universidade de São Paulo. Câmara de Fármacos e Medica- 
mentos da Subcomissão de Avaliação Terapêutica da Comissão de Avaliação Tecnológica em Saúde da Diretoria Clínica e Divisão de Farmácia. Guia Farmacoterapêutico HC 2002-2003. São Paulo: HC; 2002.

36. Hospital Geral Dr. Waldemar de Alcântara. Formulário para solicitação de alteração na lista de padronização de medicamentos [online]. [acesso em 10 fev. 2010]; 2004. Disponível em www.isgh.org.br/download/farmacia/regimento_interno.pdf.

37. Wettermark B, Haglund K, Gustafsson LL, Persson PM, Bergman U. A study of adherence to drug recommendations by providing feedback of outpatient prescribing patterns to hospital specialists. Pharmacoepidemiol Drug Saf. 2005;14(8):579-88.

38. Fijn R, Lenderink AW, Egberts AC, Brouwers JR, De Jong-Van DenBerg LT. Assessment of indicators for hospital drug formulary non-adherence. Eur J Clin Pharmacol. 2001;57(9):677-84.

39. World Health Organization. ATC Index with DDD 2008 [online]. [acesso em 10 fev. 2009]Oslo: WHO; 2008. Disponível em http://www.whocc.no/atcddd/.

40. Ministério da Saúde. Secretaria de Ciência, Tecnologia e Insumos Estratégicos. Departamento de Assistência Farmacêutica e Insumos Estratégicos. Relação Nacional de Medicamentos Essenciais. Brasília: Ed MS; 2007.

41. World Health Organization. WHO model formulary 2006 [online]. [acesso em 10 fev. 2009] Geneva: WHO; 2006. Available from: http://mednet3.who.int/EMLib/modelFormulary/modelFormulary.asp.

42. Thomson Micromedex. Micromedex healthcare [online]. [acesso em 10 fev. 2010]. Available from: http://www.periodicos.capes.gov.br

43. Agência Nacional de Vigilância Sanitária. Bulário Eletrônico da ANVISA [online].[acesso em 10 fev. 2009]. Disponível em: http://www.bulario.bvs.br

44. Ministério da Saúde. Banco de Preços em Saúde (BPS) [online]. [acesso em 10 fev. 2009]. Disponível em: http:// www.bpreco.saude.gov.br

45. Dicionário de Especialidades Farmacêuticas (DEF) 2007/2008. Rio de Janeiro: Ed Publicações Científicas; 1996.
46. Associação Brasileira do Comércio Farmacêutico. Revista ABCFARMA. Disponível em: http://www.visaocomunicacao.com.br.

47. Carvalho PRA, Carvalho CG, Alievi PT, Martinbiancho J, Trotta EA. Identificação de medicamentos "não apropriados para crianças" em prescrições de unidade de tratamento intensivo pediátrica. J. Pediatr. 2003;79(5):397-402.

48. Laing R, Tisocki K. How to develop a national formulary based on the WHO model formulary: a practical guide. Geneva: WHO; 2004.

49. Organização Mundial da Saúde. A importância da Farmacovigilância: monitorização da segurança dos medicamentos. Brasília: OPAS; 2005.

50. Brasil. Agência Nacional de Vigilância Sanitária. Resolução RDC no 220 de 21 de setembro de 2004. Aprova o Regulamento Técnico de funcionamento dos Serviços de Terapia Antineoplásica. Diário Oficial da União. Brasília, 23 set 2004; Seção 1, p. 72.

51. Ministério da Saúde. Secretária de Assistência a Saúde. Protocolos clínicos e diretrizes terapêuticas: medicamentos excepcionais. Brasília: MS; 2002.

\section{CONTRIBUIÇÃO DOS AUTORES}

Rachel Magarinos-Torres foi responsável pela coleta das informações, análise e redação da primeira versão do artigo. Dante Pagnoncelli aportou conhecimentos relacionados aos antineoplásicos. Almiro Domiciano da Cruz Filho contribuiu na discussão dos aspectos gerenciais e da prática clínica em Pediatria. Claudia Garcia Serpa Osorio-de-Castro colaborou na discussão dos achados, redação e revisão final.

\section{CONFLITO DE INTERESSES}

Declarou não haver.

\section{ENDEREÇO PARA CORRESPONDÊNCIA}

Rachel Magarinos-Torres

Rua Aguiar, 21 — apto 402

Tijuca - Rio de Janeiro

CEP 20261-120 - RJ

E-mail: rmtorres@infolink.com.br 